\title{
A Hospital Based Study of Pulmonary Tuberculosis and HIV Co-Infection - In North India
}

\author{
Vikas Kumar, Arti Agrawal, Namita Srivastawa, Ankur Goyal and Richa Gupta*
}

Sarojini Naidu Medical College, Agra, Uttar Pradesh, India

*Corresponding author

\begin{tabular}{|l|}
\hline Ke y w o r d s \\
Pulmonary \\
tuberculosis, HIV, \\
Co-infection, \\
NACO \\
\hline Article Info \\
\hline $\begin{array}{l}\text { Accepted: } \\
16 \text { April } 2018 \\
\text { Available Online: } \\
10 \text { May } 2018\end{array}$ \\
\hline
\end{tabular}

\section{Introduction}

Tuberculosis continues to remain a serious public health problem of the developing world. It is the most prevalent infectious cause of human suffering and death worldwide (Zumla et al., 2001). TB shortens the survival of patients afflicted with HIV infection, may accelerate the progression of HIV and is the cause of death in one third of people with AIDS worldwide. The higher mortality is due to the progression of AIDS rather than TB probably due to the fact that $M$. tuberculosis
India has a high tuberculosis burden and it remains one of the significant contributor and major cause of death in patients living with HIV. Tuberculosis can occur at any stage of HIV disease, and its manifestation depends largely on the level of immunosuppression. With the expansion and convergence of the HIV and tuberculosis epidemics worldwide, clinicians will increasingly be called on to manage and treat co-infected patients. The objective of this study was to determine the prevalence of pulmonary tuberculosis and associated HIV infection among suspected pulmonary tuberculosis patients attending at our hospital. This study covering the period from January 2017 to June 2017 was conducted at Department of Microbiology, SN Medical College, India. A total of 300 patients suspecting of having tuberculosis and consenting for HIV testing were included in the study. The TB diagnosis was performed using sputum microscopy (AFB staining) and all the cases were screened for HIV by using rapid HIV kits according to NACO guidelines. Overall, $29(9.66 \%)$ cases were diagnosed as sputum positive pulmonary tuberculosis among which 2 cases $(6.90 \%)$ were sero-positive for HIV antibodies. HIVTB co infection represents a novel pathogenic scenario at the global level. It constitutes a serious diagnostic and therapeutic challenge in poor countries. In our study, the prevalence of pulmonary TB was $9.66 \%$ and HIV_-TB co-infection was $6.90 \%$. increases viral replication (Swaminathan and Narendran, 2008). While HIV and TB can individually be the major causes for public health threats, the combination of the two has proven to have a far greater impact on the epidemiologic progression and consequently on the global health scene. The dual infection has been termed "the cursed duet" (Jaiswal et al., 2012). A significant percentage of tuberculosis patients are HIV positive. According to World Health Organization (WHO, 2010) global TB report, the annual incidence of TB was 9.4 million cases, of 
which 2 million cases were from India. India is 17th among the 22 countries with the highest TB incidence rates. The global list of 41 countries with the highest HIV-TB burden includes India, Indonesia, Myanmar and Thailand in Asia and the Pacific region (HIV Surveillance, 2006). The problem is now further complicated by relentless spread of HIV which causes AIDS pandemic and the emergence of multi-drug resistant strains. HIV infected individuals co-infected with tuberculosis have an annual risk of $5-15 \%$ of developing active tuberculosis (HIV Surveillance, 2006). The WHO/IUATLD global project on anti-tuberculosis drug resistance surveillance $1994-1997$ has reported $6 \%$ median sero-prevalence of HIV co infection among TB patients. (WHO, 1996) In India, tuberculosis is the most common opportunistic infection among HIV seropositive patients (Swaminathan et al., 2000). The prevalence of pulmonary tuberculosis and TB - HIV co infection is variable and periodic estimates of the same help in assessing the disease burden in that part of the country and in effective implementation of the control strategies. In India, information on co-infection remains sparse. Reports from the cities/states of India are available and indicate prevalence ranging from $<1 \%$ to approximately $30 \%$.Information about the HIV-TB co-infection in pulmonary tuberculosis patients is essential for responding with an increasing commitment to providing comprehensive HIV/AIDS care and support, including anti-retroviral therapy (ART), to HIV-positive TB patients. Therefore, this study was designed to study the prevalence of HIV-TB co-infection in patients of pulmonary tuberculosis.

\section{Materials and Methods}

This observational study was conducted from January 2017 to June 2017 for the total period of Six months at Microbiology Department
S.N Medical College, Agra. A total of 300 clinically suspected cases of tuberculosis were screening for pulmonary TB constituted the subjects for study. The diagnosis of tuberculosis was performed using clinical examination and by Acid Fast staining technique. All sputum positive pulmonary tuberculosis cases were further tested for HIV testing by using blood samples obtained from willing patients. The blood samples of all the pulmonary tuberculosis patients were then tested for HIV $1 \& 2$ antibodies following the NACO guidelines (NACO guidelines for HIV testing).

\section{Results and Discussion}

A total of 300 patients were screened for pulmonary tuberculosis at designated microscopy centre by examination of sputum samples by $\mathrm{ZN}$ staining. Among these 300 patients, there were 186(62\%) males and $114(38 \%)$ females. Clinical presentations of total patients are shown in (Table 1). Age group distributions of total tuberculosis positive patients are shown in (Table 2). Among the patients screened, 29(9.66\%) cases were found positive for tuberculosis by microscope and among these tuberculosis patients, a total of $2(6.90 \%)$ cases tested positive for HIV antibodies by serology. Tuberculosis remains to be major public health concern despite the existence of antitubercular drugs and more than a million people die of the disease every year (Bhagyabati Devi et al., 2005). Surveys in Asia and Africa have indicated that the prevalence of HIV among tuberculosis patients is much higher than that observed in the general population (Narain and Ying-Ru Lo, 2004). HIV-TB co-infection is on the rise more so in the developing countries like India, such as Mumbai (30\%), Pune (28.75\%) and Mangalore $(21 \%)$, com-pared with areas with a low prevalence, such as New Delhi (0.68\%), J \&K (1.6\%), Aligarh (2.8\%). 
Pie diagram.1 Clinical presentation of suspected tuberculosis patients

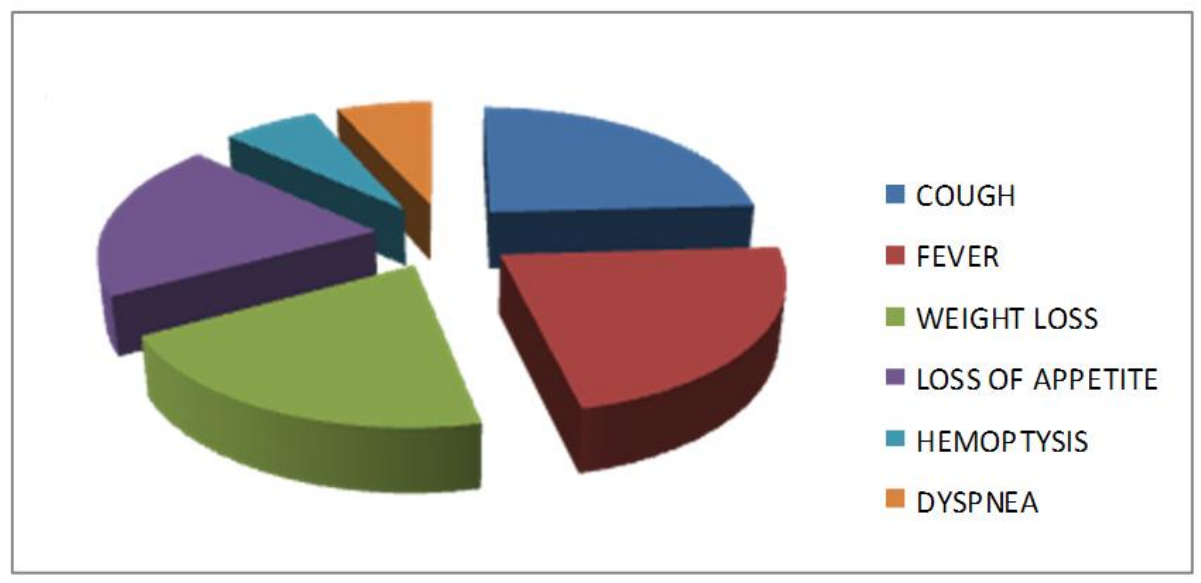

Graph.2 Age distribution of tuberculosis positive patients

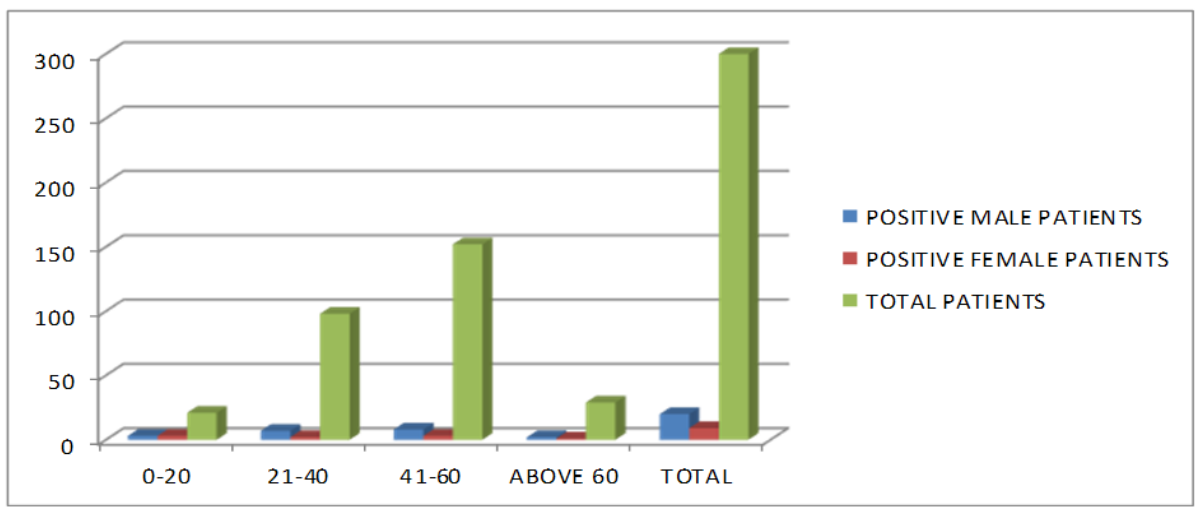

Table.1 Clinical presentation of suspected tuberculosis patients

\begin{tabular}{|c|l|c|}
\hline S. no & \multicolumn{1}{|c|}{ Symptoms } & Percentage \\
\hline $\mathbf{1}$ & Cough & 87 \\
\hline $\mathbf{2}$ & Fever & 81 \\
\hline 3 & Weight loss & 74 \\
\hline 4 & Loss of Appetite & 71 \\
\hline 5 & Hemoptysis & 24 \\
\hline 6 & Dyspnea & 24 \\
\hline 7 & Chest pain & 11 \\
\hline
\end{tabular}

Table.2 Age group distribution of tuberculosis positive patients

\begin{tabular}{|l|l|l|}
\hline Age group (yrs) & Male & Female \\
\hline $\mathbf{0 0 - 2 0}$ & 03 & 01 \\
\hline $\mathbf{2 1 - 4 0}$ & 07 & 04 \\
\hline $\mathbf{4 1 - 6 0}$ & 08 & 03 \\
\hline $\mathbf{6 1}$ \&above & 02 & 01 \\
\hline Total & 20 & 9 \\
\hline
\end{tabular}


There was a trend of increasing HIV prevalence in TB patients in Aligarh (from $0.8 \%$ to $2.8 \%$ ) from1996 to 2001 (Ahmad et al., 2003). A similar result was observed in Goa, indicating a rising trend in HIV prevalence from $2.01 \%$ in 1995 to $10.91 \%$ in 2000 (Fernandes et al., 2002). The present study indicates that there has been a low prevalence of HIV-TB co-infection (6.45\%). This change in prevalence of co-infection might be the result of strong implementation of Government programmes like RNTCP, improvements in public awareness and better treatment regimes.

This study was conducted on a limited number of sample size with in a period of six months. HIV and TB co-infection represents a novel pathogenic scenario at the global level. The impact of dual infection of HIV and TB on the economy and public health is enormous with increased morbidity and mortality. Screening of all T.B patients for HIV and vice versa will help in early detection and initiation of appropriate treatment at an early stage thus reducing the mortality rate.

\section{References}

Ahmad Z, Bhargava R, Pandey DK, Sharma $\mathrm{K}$. HIV infection sero-prevalence in tuberculosis patients. Indian Journal of Tuberculosis 2003; 50: 151-4.

Bhagyabati Devi, S., Santa Neorem, T. Jeeten Kumar Singh et al., HIV \& TB Coinfection. JIACM 2005, 6 (3); 220 -3.
Fernandes L, Lawande D, Mesquita AM. Prevalence of Human ImmuneDeficiency virus infection in tuberculosis patients in Goa. Indian Journal of Tuberculosis 2002; 49: 2356.

HIV Surveillance among Tuberculosis Patients in the South- East Asia Region World Health Organization Report, New Delhi; 2006. Pp. 11-34.

Jaiswal RK, Srivastav S, Mahajan H. Socio demographic profile of TB-HIV coinfected patients in Bundelkhand Region, Uttar-Pradesh. Natl J Med Res. 2012; 2: 149-51.

NACO guidelines for HIV testing.

Narain JP, and Ying-Ru Lo. Epidemiology of HIV_-TB in Asia. Indian Journal of Medical Research 2004; 120: 277-89.

Swaminathan S and Narendran G. HIV and tuberculosis in India. J Biosci. 2008; 33(4): 527-537.

Swaminathan S, Ramachandran R, Baskaran G, Paramasivan CN, Ramanathan U, Venkatesan $\mathrm{P}$ et al., Development of tuberculosis in HIV infected individuals in India. Int J Tuberc Lung Dis 2000; 4: 839-44.

WHO - TB group at risk: WHO report on the global Tuberculosis epidemic 1996. WWW.Who int/gtb/Publications/Therp $-96$.

Zumla A, Malon P, Henderson J. Impact of HIV infection on tuberculosis. JAMA 2001; 4:33-40.

\section{How to cite this article:}

Vikas Kumar, Arti Agrawal, Namita Srivastawa, Ankur Goyal and Richa Gupta. 2018. A Hospital Based Study of Pulmonary Tuberculosis and HIV Co-Infection - In North India. Int.J.Curr.Microbiol.App.Sci. 7(05): 2152-2155. doi: https://doi.org/10.20546/ijcmas.2018.705.250 